\title{
Quantitative Study of Positive and Negative Masculinity in Literary Works
}

\author{
Xun $\mathrm{Wu}^{1, \mathrm{a}}$ \\ ${ }^{1}$ National Institute of education, Nanyang Technological University, Singapore \\ a540164831@qq.com
}

\begin{abstract}
This paper discusses the connotation of masculinity, divides its advantages and disadvantages, and discusses its different values in different societies. This paper analyzes the degree of masculinity of characters in literary works by quantitative analysis and compares the decisive factors of masculinity in literary works.
\end{abstract}

Keywords: masculinity, Quantitative analysis, Literary works, Psychological Evaluation, eco-ethical criticism

\section{文学作品中积极消极男性气质的定量研究}

\author{
册循 ${ }^{1,}$
}

\author{
${ }^{1}$ 新加坡南洋理工大学国立教育学院, 新加坡 \\ a540164831@qq.com
}

\section{摘要}

本文对男性气质的内涵进行讨论，并划分其特征的优劣性，探讨其在不同社会中的价值。通过建构在伦理与 道德上的文学现象, 研究文学中的伦理因素, 探讨社会环境对男性气质带来的影响。结合文学与心理测评理 论, 利用定量的方式分析评价文学作品角色的男性气质, 以此为文学评论中男性气质的多寡进行多层面的定 量分析做贡献，从而直观鉴赏文学作品的男性形象的男性气质水平，实现学科交叉领域理论的构建。

关键词: 男性气质; 定量分析; 文学作品; 心理测评；文学伦理学批评

\section{1. 前言}

黑格尔曾云: “美是理念的感性呈现”，康德曾 说：“美是无目的的合目的性”。“1关于男性气质的 研究, 在不同的文本中均有体现。而何为男性之 美，也尚未有一个严格和统一的定义。但是，不同 的时代和不同的社会都给与了一定的外界评价标准 和主流的要求。本文基于人才素质测评原理和伦理 学的混合角度分析不同社会不同时代的文学作品中 的男性气质。

\section{2. 男性气质的本体研究}

\section{1 男性概念的生理学基础}

男女性的差异最根本的原因是由于不同的生理 结构所导致。而古今中外都普遍认可的观点就是男 性更适合做高强度的体力劳动，而女性则更加适合 做轻体力的工作。所以，本文选择强健的体魄作为 一个男性气质的重要标准。

“巨人” 作为人的体力的最高拥有者, 在古代 的中外文学都有存在的痕迹。在我国的《史记》、 《汉书》和《新唐书》中都出现过。传说后稷的母 亲姜嫄便是因为践踏巨人的足迹而怀孕，生下了后 稷。“后稷母为姜嫄, 出见大人迹而履践之, 知于 
身, 则生后稷。姜嫄以为无父, 贱而弃之道中, 牛 羊避不践也”, ${ }^{221}$ 表明了古代时代中对体型巨大的人 的崇拜。

西方英国的著名民间故事《杰克与豆茎》和歌 革与玛各故事都是普通人战胜并俘虏巨人的故事 ${ }^{\mathbf{3 1}}$ 以及英国作家斯威夫特《格列佛游记》中自私的巨 人的描写。 ${ }^{[41}$ 这里通过对比中外, 可以得出, 中国 对强者持有一种崇拜的观念, 而西方则是反抗精神 和自我观念强烈的观点和意识, 这也正是他们的精 神价值观的不同，形成不同的民族性格，也造成了 后期西方国家对东方的常年对外侵略与无情掠夺战 争史。

对外貌的认知, 普遍都对肌肉孔武有力、身材 高大、长相英俊的男性持有更加男子气质的评价。 无论是当代西方世界中中上层阶级对健身的喜爱, 还是东方世界中对男子外表的评价, 这个标准都趋 向于一致。

但在对男性气质的评价上, 外表和内心的评价 权重比较上中, 心理气质相比外貌更加重要。

\section{2 男性气质的生理和心理关系}

通常文学作品中将男性气质的生理特征即外 貌、行为动作特征以及声音等和该形象的心理特征 也就是性格相一致, 这样的布局给读者的非常协调 的感觉, 且常符合读者心理预期的形象构建。

《水做传》中的梁山好汉形象是如此, 个个都 威武高大, 疥恶如仇 ${ }^{51}$ 。 《穆斯林葬礼》中的青年教 师楚雁潮形象, 也是如此。其酷似学生的样貌为他 对学术研究的初探的热忱以及和女大学生韩新月的 爱情悲剧埋下了伏笔 ${ }^{\mathbf{6} 1}$ 。

此外, 也有另一种男性形象, 其生理和心理特 征呈现反差, 给读者一种独特的阅读体验, 一般都 为配角居多, 有时候会起到幽默或者反讽的效果。该 形象主要是负责进行夸张的人物形象的呈现。如

《夏夜知君暖》中的青梦篮球队员——齐峰, 就是 强壮的大块头形象, 一般文学作品中这样的身材通 常设定的性格都是攻击性强的性格, 但是该人物的 性格却出乎意料的老实和好脾气, 被人称为 “铁㷫 㮩”, 该人物的故事情节充满了喜剧和幽默的反差 感, 给读者印象深刻。

\section{3 男性概念的心理学研究}

相对而言, 男性的心理学概念包括的是与女性 气质不同的、体现男子气概性格的高水平。和外貌 不同, 男性的心理特征与所处的社会有着很强的联 系, 随着社会的演进而发生一定的改变。

但是, 部分性格特征却比较稳定: 如 “勇敢” “敢于担当” “情绪的稳定性高” 等性格特质。

大五人格理论是心理学家图普斯、克洛斯特
尔 (Tupes \& Thristal) 发现的五个显著而又稳定 的因子, 后其他心理学家在此基础上提出了外倾 性、宜人性、责任心、神经质和开放性五大维度 [7]。

在这些参考指标中根据重要程度加入权重系 数。

最被公认的男性气质的考察指标为一一责任心 指标, 属于关键性指标。

其次是一一情绪的稳定性, 也就是具备稳定的 性格, 神经质特征的反面特征。通常认为可以稳定 处理情绪的能力属于男性特质, 即: 可以平衡焦 虑、敌对、压抑的情绪, 具备高强的抗压能力的品 质。包括 “男子有泪不轻弹” 等经典语句。而情绪 易于波动、抑郁等情绪则被认为是女性化的气质。

宜人性在目前的社会至关重要, 但是随着社会 场合的不同也会出现不同变化。在和平的年代, 宜 人性和西方的绅士风度有相似之处, 前者为性格, 后者为行为。

越高的宜人性越表明越高的绅士风度, 在长远 上看，也有利于社会的稳定和谐。而较低的宜人性 属于男性的暴力倾向及支配情绪, 属于物质资源嘈 乏的社会中需要进行物质争夺时期男性气质的范 畴, 但是一定程度不被当今社会所提倡, 除了扩张 和保护自己的领地的时候, 此时的斗争性可以使得 男性所代表的整个种族群体获得更佳优质的生活、 生存环境和条件。如火到全亚洲的《流星花园》中 的男主角道明寺 ${ }^{81}$, 他的攻击性和霸道性格颇受到 观众的喜爱; 钢铁侠等英雄人物至今也都是当代男 性的偶像, 每次的电影的销量都是稳居男性最爱电 影榜首。由此可见, 冲击性高的男性气质在男性群 体中尤为重要, 基本属于地位的体现。

外倾性: 中西方中人与人相处都相比较喜欢乐 于交际、乐于沟通的男性形象。

但评价标准则主要根据不同的所处环境而改 变。在商业领域, 外向的性格与积极主动的个性特 征广为推崇, 因为商业活动中优秀的交际能力和交 际意愿是提升其管理技能的关键，欧美国家的商科 就业主要是依据朋友介绍; 但是, 科技界和学术界 的人来说, 内倾型性格也便于其扎根学术研究中。 一般而言, 中高强度的外向型最受推崇, 既保持了 人与人之间的联系与沟通又满足了彼此之间的合理 的交际边界, 且中高强度的外向性格可以使得其研 究成果和名声等得以传播, 从而扩大影响力。相 反, 较为内向的人一般较不易于被人了解, 也不利 于社交圈的扩大，不适合地位的提升。

开放性方面, 一个人思维的开阔性、好奇心和 深刻的原创性程度。一般没有非常明显的倾向, 主 要依照不同的时代和岗位而有所区分。总体上，随 着工业和科技革命时代的到来, 创新被推崇, 尤其 是当今世界的商业和科学领域, 高开放性至关重 
要; 但是, 低开放性也很重要, 在传统的办公室的 工作岗位中，低开放性可以保证其工作的稳定性。 时代不同，需求也不同，在航海时代，开放性强的 男性意味着更强的意愿去开拓新领土, 具备更强的 冒险精神, 大体上是值得被推崇的; 而中国的农耕 经济, 则需要一定的低开放性特质来帮助其继承先 辈的土地, 从而进行稳定的耕种工作。开放性这个 特质的高低程度和男性气质关系不太大。

按照个性特征的重要程度进行权重的赋值, 将 特征的程度与男性气质计分, 将责任心权重计为 3 , 神经质性计为 2 , 外倾性记为 1 , 计算平均分。 越接近于 10 , 则表示男性的性格气质越高, 见表 1 。

\section{3. 男性气质的中西对比}

\section{1 中国的男性气质}

\subsection{1 中国古代的男性气质}

中国古代文学中男子的强烈气质主要是男尊女 卑、“学而优则仕” 以及部分反抗英雄对腐败的朝廷 的抗争行为的体现。

古代文学作品中对不懂政治只喜欢艺术文学的 男子，所持批评、扼腕的态度居多。如 “为宰相而 作小词, 可乎? ”一—魏泰《东轩笔录》。【9】 “郑卫 之音, 乱世之音也, 亡国之音也”-—诗经. 国风。

且如后主李煜痴迷于诗词, 朝廷重文轻武的政 策, 导致宋代朝廷的衰败。

甚至古代的小词体裁也多为表示男女感情结合 配乐写成, 词主要用男女情感借比朝廷的统治。“词 之雅郑, 在神不在貌。永叔、少游虽作艳语, 终有 品格”-—王国维《人间词话》也体现出其主要以 写爱情为形来壮志 ${ }^{[11]}$

《红楼梦》中贾宝玉形象, 一个古代封建大家 族的得宠的公子哥, 沉迷于男女之情感, 不钟情于 仕途, 最后整个四大家族衰败的结局, 体现了古代 男子应该以仕途为重的思想和古代社会明显的等级 秩序 ${ }^{\mathbf{1 2}}$ 。

《三国演义》中的关云长刮骨疗毒, 见小说第 七十五回。其中主要通过描写其他人的 “掩面失 色” 的神态和描绘刮骨疗毒的治疗方法的残忍来对 比凸显出关云长神情的淡定, 体现英雄的勇敢和神 威 ${ }^{[131}$

\subsection{2. 现代中国的男性气质}

从鸦片战争开始, 中国社会的面貌发生了很大 的变化, 中国文学受到西方文学的巨大影响, 部分 学者甚至主张全盘西化。
西方文化中尤其是英国的上层的绅士文化被中 国当时的先进知识分子所推崇。部分有钱人家的男 子开始流行海外留学且当时思想家和作家是社会革 命的主力军, 所以他们所推崇的文化也被整个社会 所崇拜与向往。于是，文人的谦谦君子形象作为一 种主流的男性气质开始登上历史舞台。

东方的爱情小说, 如张爱玲的《红玫瑰与白玫 瑰》一文中将男主人公写成了朝秦暮楚, 没有真正 唯一挚爱的男性, 而女性形象也都是一些负面的形 象出现, 或多或少有着一些缺陷, 甚至存在婚内出 轨等问题。这也一定程度上反映了当时包办婚姻的 不幸以及自由恋爱开始时期男性对待女性的态度、 反映了社会重男轻女的社会风气，揭示了男性选择 配偶时的一些社会中普遍被认可的标准：长相清 秀、脾气温柔、交际圈小、家世清白等 ${ }^{114}$ 。

\section{1.3 当代中国的男性气质}

随着人工智能和知识化、信息化时代的到来, 一些重体力劳动已经被机械所取代, 越来越多的岗 位需要凭借细心和严谨、认真的性格才可以胜任。 且随着媒体的传播, 西方的女权运动也逐渐影响到 了中国等东方国家, 女性地位逐年上升, 甚至出现 了女性对男性的消费, 对于男性气质的审美变得逐 渐多元。

但是, 主流的新闻媒体依然主张过去的男子气 概，对于当下年轻群体的审美追求持有一定的否定 态度, 这也表明了被推崇的男子气质在不同的时代 具有较为稳定的个性特征。

从 21 世纪 10 年代开始, 韩国的 EXO 偶像组合 风靡全中国乃至亚洲和全球, “小鲜肉” 成为 90 后 00 后部分女性疯狂崇拜的对象, 受到了部分年纪较 小的女生的喜欢。但是，从“小鲜肉”这一名词也 可以得出其男性气质偏低龄，不属于严格意义上被 大家和社会认可的成熟男性气质。此外, 这些形象 一直饱受争议, 受到了很多的负面的评价, 经常将 这些偶像评价为女生，甚至 “娘炮” 等。中国的

《人民日报》提出关于 “小鲜肉” 的言论, 其主要 观点为外表不是最主要的, 内心和努力的成果才是 关键指标，也可以看出男性气质评价的依据和重点 所在, 此外对于这种现象也进行了一定的抵制 ${ }^{\mathbf{1} 15}$ 。

截至 2019 年上半年中国手机网络文学用户规模 达 4.35 亿人，网民使用率为 $51.40 \%$ 。网络文学用 户男女比例分别为 56.4\%和 $43.7 \%$, 热门小说排行榜 中，盗墓、探险、玄幺修真题材网络文学作品的排 名均靠前, 选择排行榜最高的 “起点中文网” 的玄 幻小说，可见人气最高的小说名有 “诡秘之主” “完 美世界” “斗罗大陆 IV 终极斗罗” 等, 受到男性读 者更多的偏爱。【16】而这些的主人公多属于生下来就 拥有绝世武功以及面容姣好之男性, 或者是从一个 普通人逆袭到一个力量无边的男子形象。这样的主 人公形象的建构满足了当代中国男性读者的自我期 
望形象的寄托和影射，即，成为更具备理想男性气 概的人。乌托邦的男性形象的构建符合当下读者对 自身现实形象的不满和解脱, 中国当今社会的压力 与日俱增, 人均生活水平差异大, 而网络文学的理 想人物的建构寄托着男性对自身的形象的期盼和寄 予。

\section{2 西方的男性气质}

\subsection{1 中世纪之前的男性气质}

尤其是在古罗马、古希腊时代，主要强调勇敢 这一特性。当时的西方世界主要为沿海的国家，陆 地面积比较狭小, 不能实现自给自足的农业生活, 所以商业生活尤为频繁, 每天面对着大海的他们, 也想去扩张市场, 于是远洋航行和战争对他们而 言, 非常重要。而这些活动的实现, 又都需要勇敢 作为支撑。

\subsection{2 当代西方的男性气质}

英国根据不同的阶级分为了欧洲贵族的绅士气 质, 中产阶级的追求资本的个性特征以及底层劳动 人民的集体性的性格特质。其主要划分依据是目前 英国甚至整个西方世界都属于资本主义社会, 人与 人之间的区别主要在于金钱的拥有量, 而不同层级 的人为了彼此区分和更好的适应其所在的社会的角 色, 选择了不同的处事方式为自己的行为准则, 长 期就形成了三种不同的风格类型。

而东西方对待爱情则不同, 尽管 18 和 19 世纪 女性文学仍然被看做是隶属于男性活动的公共领 域。但是总体上, 西方的文学小说中女性地位仍然 较高, 仅仅是 19 世纪就涌现出了 30 多位有影响力 的著名女作家, 作为解构者, 把文学作为解构男性 为中心的途径, 宣扬追求自己的爱情, 而不是沉浸 于失去爱情的苦痛之中, 主要将男女的爱情视为积 极正面的感情, 而非是不当关系。《简爱》则是描绘 一个女性面对一个外貌、地位都比自己优秀的男性 的爱情的时候的平等的态度和伦理诉求。此类作品 不乏对爱情的描写与分析：司汤达的《红与黑》、 《傲慢与偏见》等。

\section{3 负面男性气质}

\subsection{1 中国的负面男性气质}

鲁直笑曰: “空中语耳, 非杀非俯, 终不至坐此 邽恶道”, 在过去社会的道德价值观中是燋不上作奸 犯科以及沉溺于爱情的男子的 ${ }^{[171}$ 。早年也有对不忠 不义之人的批判, 如陈世美被文学典型秉公执法人 物包拯所斩。

\section{3. 2 西方的负面男性气质}

负面的男性气质多为虚伪, 作奸犯科等品质。 该类别的性格具备普适性, 古今中外对其评价差别 较小, 都持否定态度。如彼得凯里的《销售边缘气 质》便是如此 ${ }^{\mathbf{1 1 8 1}}$ 。

\section{4 中西方世界男性气质的异同}

\subsection{1 中西方男性气质的相同点}

中西方男性气质的本质为有力量的有实力的可 以保护家庭, 推动社会发展, 赢取更多的财富和地 位的外貌特征和个性特征。

双方都唾弃并摒弃虚伪的圆滑油淢的男子形 象。

\section{4.2 中西方男性气质的不同点}

由于西方社会中集会运动的普遍性, 使得女权 运动较为盛行, 且有统计数据表示, 西欧国家的女 性地位较高, 而日本的女性地位较低。这也导致了 西方世界的上流社会更倾向于认为绅士气质为上流 社会的男性气质, 将粗鲁视为下层以及蓝领阶层的 性格特质; 而现代的东亚社会仍认为霸道、血性为 男性气质的表现。

\section{4. 男性气质和社会阶层的关系}

男性气质和社会阶层中的关系也较为清晰。隋 红升指出, 西方世界主要依据阶层划分了三种层次 的男性气质, 每个阶层有着其独特的伦理追求 ${ }^{[191}$ 。 上流阶层主张儒雅、智慧有气质; 中层阶级主张男 性拥有超强的能力和竞争力; 而底层社会则是主张 血性、粗鲁和掌握一门手工技能。根据阶层划分的 优质男性气质，体现了男性作为一个 “经济人” 的 无奈。男性角色为了活得更好, 不得不为了生存而 努力, 从而被他人打上了他所在阶级优秀特征的烙 印。似乎他满足了这样的特征, 他就是这个阶层最 具备男性气质的人, 最具备竞争力的人, 全然忽视 了男性自身的内心意愿与心理健康及心理归属。

男性也逐渐被物化。全世界享受着飞速发展的 物质生活的同时, 全球竞争加剧, 职场人的精神压 力越来越重, 男人作为传统的应当支撑起家族的角 色, 肩负着更沉重的责任。职场中自杀、过劳死等 恶性事件层出不穷, 而社会却缺乏对他们的关照和 重视。中层阶层的西方男子为了获得更好的学校的 青睐, 或是为了积累更雄厚的人脉, 不得不具备超 强竞争力与实力; 底层男性的手艺技术高超如何直 接影响了他的劳动价值, 也就是工资。每个男性都 被物化, 价值化。西方如此, 中国亦然。

一般而言，上层等级拥有更多的偏爱权和被保 护权利。底层的男子多充当一种利用自身优势而保 
护上级的保护者形象, 《追风筝的人》里面哈桑, 一 个下层等级的男子都有执行保护其所侍奉的主人的 使命和任务，而主人公习惯懦弱，也仅仅是在知道 是自己同父异母的兄弟之后，才决定救赎。 ${ }^{\mathbf{2} 20}$ 正是 血缘和地位唤醒了主人挺身而出的行为, 改变了他 们的观念和行为准则。从此, 不少的读者纷纷决定 加入到反奴隶制以及关注人权的志愿行为中去。中 国古代文学《三国演义》里面对才华横溢的诸葛亮 对蜀国刘邦的支持以及刘禅的保护也都体现了统治 阶级的易受保护的特征 ${ }^{\mathbf{2} 11}$ 。

\section{5. 男性特征与人格测试的关系}

利用权重和心理测评问卷方式对男性气质进行 问卷调查和测评, 计算其男性气质得分。参照表 $1 、 2.3$ 。其中, 量表一为男性气质在个人形象中的 体现, 主要测评的为外貌和性格因素; 量表三主要 考察其在社会中的男性气质; 量表四主要考察期负 面的男性性格气质。这三者量表的结合, 分别计算 分数, 从而便于对其类型进行更精确的划分, 体现 文学人物的多面性。

表 1 男性气质与大五人格的关系

\begin{tabular}{|l|l|l|l|l|l|}
\hline & 开放性 & 责任心 & 外倾性 & 宜人性 & 神经质性 \\
\hline 弱 & & & & & \\
\hline & & 2 & 3 & & 10 \\
\hline & & 3 & 5 & & 9 \\
\hline & & 4 & 6 & & 8 \\
\hline & & 5 & 7 & & 6 \\
\hline & & 6 & 8 & & 5 \\
\hline & & 7 & 9 & & 4 \\
\hline & & 8 & 10 & & 3 \\
\hline & & 9 & 9 & & 2 \\
\hline 强 & & 10 & 8 & & 1 \\
\hline
\end{tabular}

本量表根据更为普遍的男性气质伦理的标准进 行计分。分数越高, 代表越符合大众对男性性格与 气质的评价。排分越低越被大众评价为 “缺乏男性 气质和男性气概” 或其他形容女性气质的称谓。

表 2 男性气质测评问卷表

()

1-1 身材比一般人高大。

2-1 肌肉比一般人更加壮实。 ○

3-3 一 般在困境中仍然自信。

()

(0)

4-1 长相看起来很有孩子气。

5-1 肌肉很少, 看上去没有力量。
()

6-2 言必出, 行必果, 说一不二.

7-4 一般情况下, 都比较懦弱, 不敢说出自己 的想法也不敢反抗别人

(0)

8-4 遇到别人的威胁和压迫, 选择奋起反抗。

()

9-4 很勇敢

(0)

其中，第一个数字为题号，第二个数字为题目 所考察的属性。

1 外表上的男性气质

2 责任感

3 抗压能力

4 勇敢

此外, 123689 属于加分项目。其余为减分项 目。

通过检验各题目答案是否为自相矛盾, 检验其 问卷填写的有效性。

表 3 男性成就测评量表

\begin{tabular}{|c|c|c|c|c|c|}
\hline & 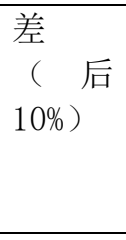 & $\begin{array}{l}\text { 较 } \text { 为 } \\
\text { 差 的 } \\
\text { 水 平 } \\
(\text { 后 } \\
25 \%)\end{array}$ & $\begin{array}{l}\text { 一般 / } \\
\text { 普 通 } \\
\text { 水平 }\end{array}$ & $\begin{array}{c}\text { 较 为 } \\
\text { 优 秀 } \\
\text { 水 平 } \\
(\text { 前 } \\
25 \%)\end{array}$ & $\begin{array}{c}\text { 非 常 } \\
\text { 优 秀 } \\
\text { 水 平 } \\
(\text { 前 } \\
10 \%)\end{array}$ \\
\hline $\begin{array}{l}\text { 财富 } \\
\text { 值 }\end{array}$ & & & & & \\
\hline $\begin{array}{l}\text { 地位 } \\
\text { 值 }\end{array}$ & & & & & \\
\hline $\begin{array}{l}\text { 智商 } \\
\text { 系数 } \\
\text { 值 }\end{array}$ & & & & & \\
\hline $\begin{array}{l}\text { 情商 } \\
\text { 系数 } \\
\text { 值 }\end{array}$ & & & & & \\
\hline 总分 & & & & & \\
\hline
\end{tabular}

评分标准：一共 100 分。各个项目各为 25 分。 其中，非常优秀水平到差依次为 25、20、15、10、 5 分。 
表 4 男性负面性格测评量表

\begin{tabular}{|c|c|c|c|c|c|}
\hline & $\begin{array}{l}\text { 差 } \\
(\text { 后 } \\
10 \%)\end{array}$ & $\begin{array}{l}\text { 较为差的 } \\
\text { 水平 (后 } \\
25 \% \text { ) }\end{array}$ & $\begin{array}{l}\text { 一般/ } \\
\text { 普通水 } \\
\text { 平 }\end{array}$ & $\begin{array}{c}\text { 较为优 } \\
\text { 秀水平 } \\
(\quad \text { 前 } \\
25 \%)\end{array}$ & $\begin{array}{c}\text { 非常优 } \\
\text { 秀水平 } \\
(\quad \text { 前 } \\
10 \%)\end{array}$ \\
\hline 虚伪 & & & & & \\
\hline $\begin{array}{l}\text { 阿 妷 } \\
\text { 奉承 }\end{array}$ & & & & & \\
\hline $\begin{array}{l}\text { 忘 恩 } \\
\text { 负义 }\end{array}$ & & & & & \\
\hline $\begin{array}{l}\text { 沉 迷 } \\
\text { 酒色 }\end{array}$ & & & & & \\
\hline $\begin{array}{l}\text { 其 他 } \\
\text { 负 面 } \\
\text { 性 格 } \\
\text { 特征 } \\
\end{array}$ & & & & & \\
\hline 总分 & & & & & \\
\hline
\end{tabular}

()

1-1 言而有信。

2-1 说出的话与实际的行为并不一致。 ()

3-3 面对利益的时候，友谊一文不值。 ()

4-2 对上层进行贿赂。 5-1 很少撒谎。

(C)

6-2 面 对上 司，进 行 虚假的 讨 好。

7-4 喜 欢逛酒吧和夜店

8-4 不喜欢喝酒和出入娱乐场所。 ()

9-3 友谊, 兄弟义气非常珍贵 ())

10-4 喜欢和多位女性进行暖昧 (0)

其中, 第一个数字为题号, 第二个数字为题目 所考察的属性。

1 外表上的男性气质

通过检验各题目答案是否为自相矛盾, 检验其 问卷填写的有效性。

\section{6. 结论}

中西方的男性气质有其共同点和不同点。共通
性特征都为身形高挺，肌肉结实的外貌。内在的品 质为积极的追求更仕途和事业的成功, 终极奋斗目 标为实现自身地位的提升。将虚伪、邪恶视为负面 的男性气质，对其进行批判。

不同之处在于中国自从古代开始就将盲目追求 男女爱情和的文艺不务正业划为等号, 中国崇尚的 男性气质更加偏向保守, 外倾性水平较低; 西方男 性气质推崇更加的具有阶级性, 且更加的将爱冒险 和敢于挑战视为更加优质的男性气质。

最后, 这些研究的局限在于缺乏了对人性的关 怀和关照。

\section{REFERENCES}

[1] Zhu G.(2019) History of Western aesthetics. China Friendship Publishing Company,Beijing.

[2] Sima Qian, ed.(2016) Records of the Historian .Beijing United Publishing House, BEIJING.

[3] FairyTales,(2018).Jake and the beanstalk.https://www.sohu.com/a/235860769_703 360 .

[4] JonathanS.(2019) Gulliver's Travels.Jiangsu Phoenix Literature and Art Publishing House, Nanjing.

[5] Shi N, Luo G.(2009) Heroes of the Marshes.Zhonghua Book Company, Beijing.

[6] Huo D.(1988) a Muslim funeral. Beijing October Art and Literature Publishing House,Beijing

[7] Goldberg, L.(1990). An alternative "description of personality": The Big Five factor structure. Journal of Personality and Social Psychology, 59, 12161229.

[8] 360baike,(2018).MeteorGarden.https://baike.so.com/ doc/3998746-27429657.html

[9] Wei T, Li Y.(1983), Dong Xuan Bi Lu.Zhonghua Book Company,Beijing.

[10] Confucius (2006) the book of Songs. Beijing Publishing House,Beijing.

[11] Wang G (1998) human ci Hua. Shanghai Ancient Books Publishing House,Shanghai.

[12] Cao X, Gao E (1996) a dream of Red Mansions.People's Literature Publishing House, Beijing.

[13] Luo Guanzhong (1986), Romance of the Three Kingdoms. Yuelu Press, Changsha.

[14] Eileen C (2009) red rose and white rose.Beijing October literature and Art Publishing House, 
Beijing.

[15] People's Daily, overseas edition.(2018).Talking about the phenomenon of femininity.https://baijiahao.baidu.com/s?id=160941 4588119664842

[16] Qianzhan.com,(2020), Till the half of 2019, the number of online literature users in China reached 455

million.https://xw.qianzhan.com/analyst/detail/220/ 200309-4405e9f2.html

[17] Stendhal, Z.(2002) red and black.People's Literature Publishing House, Beijing.

[18] Peter C.(2014)marginal masculinity: A study of gender and national identity in fiction. University Press, Chengdu.

[19] Sui H. (2021) masculinity. Foreign Language Teaching and Research Press,Beijing.

[20] Khaled H. (2003) The Kite Runner, Shanghai People's Publishing House, Shanghai.

[21] Luo G.(1986) Romance of the Three Kingdoms. Yuelu Press, Changsha. 